Nervenarzt 2019 · 90:914-920

https://doi.org/10.1007/s00115-019-0773-8

Online publiziert: 16. August 2019

(c) Der/die Autor(en) 2019

\author{
Emrah Düzel ${ }^{1,2} \cdot$ Jochen René Thyrian ${ }^{3,4} \cdot$ David Berron ${ }^{1,2,5}$ \\ ${ }^{1}$ Institut für Kognitive Neurologie und Demenzforschung (IKND), Magdeburg, Deutschland \\ ${ }^{2}$ Deutsches Zentrum für Neurodegenerative Erkrankungen (DZNE), Standort Magdeburg, Magdeburg, \\ Deutschland \\ ${ }^{3}$ Deutsches Zentrum für Neurodegenerative Erkrankungen (DZNE), Standort Greifswald, Greifswald, \\ Deutschland \\ ${ }^{4}$ Institut für Community Medicine, Universitätsmedizin Greifswald, Greifswald, Deutschland \\ ${ }^{5}$ Clinical Memory Research Unit, Department of Clinical Sciences Malmö, Lund University, Lund, Schweden
}

\title{
Innovation in der Diagnostik - mobile Technologien
}

chologische „Paper-pencil“-Tests. Diese beiden Aspekte stellen zugleich auch die Kernprobleme der aktuellen Praxis dar.

\section{\) „Paper-pencil"-Tests sind zeit- und personalintensiv und haben signifikante Lerneffekte}

Die räumliche und zeitliche Bindung an eine Einrichtung beeinflusst zum einen die Validität der Messung durch die oft fremde und ungewohnte Situation und macht zum anderen wiederholte und engmaschige Messungen logistisch unmöglich. Zeitlich aufwendigere neuropsychologische Tests können meist nicht durchgeführt werden. In der Folge stehen Ärzten zur diagnostischen Einordnung nur einzelne Testwerte kurzer Screeningtests wie dem DemTect-Test [21] oder die MMSE (Mini Mental State Examination) zur Verfügung. Diese und andere „Paper-pencil“-Tests lassen sich nur bedingt wiederholt einsetzen, da sie signifikante Lerneffekte aufweisen [4] und eignen sich daher nicht zur engmaschigen Erfassung kognitiver Trajektorien. Bei Patienten mit auffälligen Testresultaten lässt sich daher die Kernfrage, ob Defizite progredient sind oft erst nach mehreren Jahren beantworten. Für eine adäquate Beratung der Betroffenen und eine sinnvolle Versorgung wäre es hingegen erforderlich, das Ausmaß der Symptomprogredienz innerhalb von Monaten zu bestimmen. Zusätzlich wird die Validität der Einzelmessungen auch durch intermittierende gesundheitliche oder lebensstilbedingte Faktoren (Stress, Schlafentzug) eingeschränkt [11]. Ebenso können auch Medikamente kognitive Defizite (iatrogen) hervorrufen [20] und die Interpretation von Einzelmessungen erschweren. Schließlich sind Screeningtests und neuropsychologische Testverfahren aufgrund ihrer fehlenden anatomischen Spezifizität für die neuronale Ausbreitung der AlzheimerErkrankung kaum geeignet, eine biologische Progression der Erkrankung von einer unspezifischen Verschlechterung durch Komorbiditäten zu unterscheiden.

Aus diesen Überlegungen ergibt sich, dass vor allem in der präklinischen und prodromalen Phase der Alzheimer-Erkrankung eine wesentliche Verbesserung der aktuellen Praxis erreicht werden kann durch:

1. eine räumliche und zeitliche Ent-

kopplung des kognitiven Assess-

ments von den Einschränkungen des

Klinik- und Praxisalltags;

2. hochfrequent wiederholte Messungen und ausführliche Erfassung unterschiedlicher kognitiver Domänen (über einen Zeitraum von einigen Wochen) zur Erhöhung der querschnittlichen Reliabilität des kognitiven Assessments;

3. eine schnelle Erfassung der Progredienz der kognitiven Defizite durch ein hochfrequentes, longitudinales Monitoring über einen Zeitraum von mehreren Monaten; 
4. eine Erfassung der anatomischbiologischen Progression der Alzheimer-Erkrankung und Abgrenzung von unspezifischen kognitiven Verschlechterungen.

Eine innovative Möglichkeit, diese Verbesserungen zu erreichen, sind mobile, App-basierte Technologien zur Testung kognitiver Defizite.

\section{Biologische Progredienz kognitiver Defizite im Verlauf der Alzheimer-Erkrankung}

Mobile, App-basierte Technologien erlauben es, neue Erkenntnisse aus der kognitiven Alzheimerforschung schneller als bisher in die Anwendung zu bringen. Somit wird es möglich, neben etablierten neuropsychologischen Tests auch innovative kognitive Tests in der Versorgung $\mathrm{zu}$ nutzen, die einen engen Bezug zur biologischen Progression der AlzheimerErkrankung im Gehirn aufweisen (z.B. [18]).

Die Alzheimer-Krankheit ist gekennzeichnet durch eine lange präklinische und prodromale Phase mit fortschreitender Amyloid- und Tau-Pathologie, Neurodegeneration und kognitiver Beeinträchtigung [13]. Während die Bestimmung der $\beta$-Amyloid- und der TauPathologie mit Liquor- oder molekularen Bildgebungsverfahren (z. B. [13, 16, $19,22])$ gut etabliert ist, bleibt die Bestimmung des Grades der kognitiven Beeinträchtigung und dessen Progredienz sehr schwierig. So spiegeln beispielsweise die Liquormessungen der Amyloid- und Tau-Pathologie nicht unbedingt den aktuellen Zustand kognitiver Defizite wider und erlauben keine hinreichend genaue Vorhersage der Progredienz kognitiver Defizite [13].

\section{》) Die sich ausbreitende Tau- Pathologie führt zur Störung des episodischen Gedächtnisses}

Die anatomische Progression der Amyloid- und Tau-Pathologie in der präklinischen und prodromalen Phase der Alzheimer-Erkrankung ist mittlerweile aus histologischen und bildgebenden Studi- en gut bekannt $[13,18]$. In den letzten Jahren konnten auch die kognitiven Prozesse entschlüsselt werden, die diesen anatomischen Regionen im Gehirn zugeordnet werden können. Die Tau-Pathologie breitet sich von perirhinalen und entorhinalen Subregionen im Schläfenlappen über hippokampale Subfelder und die Amygdala bis hin zu lateralen temporalen, frontalen und parietalen Regionen aus $[3,17]$. Im Rahmen dieser anatomischen Ausbreitung werden insbesondere Gedächtnisprozesse in Mitleidenschaft gezogen. Dabei handelt es sich vornehmlich um die Fähigkeit, sich die Identität und Form von Objekten zu merken $[2,18]$. Mit zunehmender anatomischer Ausbreitung, vor allem mit der Überlappung mit kortikaler Amyloidpathologie, wird auch das Gedächtnis für räumliche Information, wie z. B. die Form und Geometrie von Räumen, beeinträchtigt $[2,18]$. Zusammen erklären diese Defizite das Leitsymptom der AlzheimerErkrankung, nämlich eine Störung des episodischen Gedächtnisses, d. h. der Fähigkeit sich an persönliche Ereignisse zu erinnern $[6,8]$. Sie umfassen zum Teil auch Störungen der visuell-räumlichen Aufmerksamkeit, die bei Alzheimer-Patienten bekannt sind [14]. Ein Teil der Alzheimer-Patienten hat schon in frühen Stadien ausgeprägte Störungen der exekutiven Kontrolle, die durch etablierte neuropsychologische Tests wie TrailMaking und Digit-Symbol-Substitution erfasst werden können [7, 25, 31, 32].

Wie weiter unten dargestellt, lassen sich diese unterschiedlichen kognitiven Domänen mittels App-basierter Technologien die innovative kognitive Tests (z.B. Gedächtnis für Objekte und Räume) und etablierte neuropsychologische Tests kombinieren, alltagsnah, stressfrei und hochfrequent untersuchen.

\section{Leitsymptom Gedächtnis- probleme: Probleme aktueller Versorgung}

Patienten, die über Gedächtnisprobleme klagen, stellen sowohl in der primärärztlichen als auch in der fachärztlichen Routineversorgung eine große Herausforderung dar [27]. Niedergelassene Neurologen und Psychiater sind die erste spezia- lisierte Anlaufstelle für ältere Menschen, die über subjektiv empfundene Gedächtniseinbußen klagen [26]. Eine subjektive Gedächtniseinbuße kann diagnostisch unterschiedlich eingeordnet werden. Es kann sich um

- eine leichte Alzheimer-Demenz handeln,

- um eine leichte kognitive Beeinträchtigung (,mild cognitive impairment", MCI; z. B. beim Vorliegen einer isolierten Gedächtnisstörung) oder

- beim Fehlen eines MCI um eine subjektive kognitive Störung (SCI).

Hier stehen diagnostisch folgende Aspekte im Vordergrund:

1. Ist die Gedächtnisstörung objektivierbar oder handelt es sich um eine SCI?

2. Sind die Befunde bei einer objektivierbaren Gedächtnisstörung kompatibel mit einer MCI oder einer leichten Alzheimer-Demenz?

3. Ist die Symptomatik stabil oder progredient?

Obwohl standardisierte Screeningverfahren als Bestandteil des geriatrischen Basisassessments in der Routineversorgung abrechenbar sind, werden sie in der Routineversorgung nur unzureichend häufig angewendet [26, 27]. Die Ergebnisse sind häufig von der Untersuchungssituation in der Routineversorgung abhängig, nicht sensitiv und spezifisch genug und lassen ohne weiterführende, differenziertere Diagnostik keine klaren, handlungsleitenden Aussagen zu den oben genannten Fragen zu. Deshalb werden Betroffene häufig zu spät erkannt, sodass die Chance verpasst wird, durch eine frühzeitige Versorgung dem Krankheitsverlauf optimal zu begegnen [9]. Ebenso nehmen Menschen mit Verdacht auf eine Demenz weiterführende diagnostische Maßnahmen bei niedergelassenen Fachärzten für Neurologie und Psychiatrie seltener in Anspruch als zu erwarten wäre [24]. Deshalb müssen zusätzliche Wege gefunden werden, die Diagnostik in der Routineversorgung zu optimieren. Andererseits werden diagnostische, spezialisierte und kostenintensive Verfahren bei Personen angewandt, denen die Unsicherheit über 
ihre Symptome nicht genommen werden können.

In der Natur der häufig papiergestützten Verfahren liegt es ebenso, dass diese Tests für eine regelmäßige und engmaschige Verlaufsbeobachtung nicht geeignet sind. Werden diese Testungen engmaschig durchgeführt, „lernen“ die Patienten und werden in der Aufgabe immer besser, sodass diese Testungen letzten Endes an Güte verlieren. Darüber hinaus kommen z. B. im DemTect-Test Verfahren zum Einsatz (Hauptkomponente für die Gedächtnistestung ist der freie Abruf von 10 Wörtern), die keine anatomisch-biologische Beziehung zur Alzheimer-Erkrankung haben und daher trotz eines unauffälligen Testresultates keine Entwarnung geben können bzw. bei auffälligen Resultaten viele andere Erkrankungen ursächlich sein können.

\section{》) Übliche papiergestützte Verfahren eignen sich nicht zur engmaschigen (hoch-frequen- ten) Verlaufsbeobachtung}

Bei einem substanziellen Teil der Betroffenen mit MCI oder einer SCI (ca. 60-70\% bei 65- bis 70-Jährigen) liegt keine Alzheimer-Erkrankung zugrunde; sie haben unauffällige Biomarkerprofile (Tau, Amyloid) im Liquor [30]. Diese Patienten befinden sich somit nicht in einer frühen Phase der AlzheimerErkrankung, sondern haben kognitive Probleme aufgrund anderer Ursachen, wie metabolische oder vaskuläre Störungen, einer Depression oder einer anderen neurodegenerativen Erkrankung.

Für Hausärzte und Fachärzte gibt es derzeit keine geeigneten Verfahren, die beim Vorliegen subjektiver Gedächtnisbeschwerden eine kostengünstige und unter einem geringen Ressourcenaufwand erreichbare Einordnung in die o.g. Diagnosen erlauben und helfen können, beim Vorliegen einer SCI oder eines MCI die Wahrscheinlichkeit einer prädementiellen Alzheimer-Erkrankung einzugrenzen [10]. Zudem fehlen geeignete Verfahren, um die Progredienz der kognitiven Störungen einzuordnen.

Nervenarzt 2019 · 90:914-920 https://doi.org/10.1007/s00115-019-0773-8

(C) Der/die Autor(en) 2019

\section{E. Düzel · J. R. Thyrian · D. Berron}

\section{Innovation in der Diagnostik - mobile Technologien}

\section{Zusammenfassung}

Hintergrund. Progrediente kognitive

Einbußen sind das klinische Leitsymptom der Alzheimer-Erkrankung. Die genaue Erfassung kognitiver Defizite und die Beurteilung ihrer Progredienz bereiten jedoch in der Versorgung und Frühintervention große Probleme.

Fragestellung. Welche Probleme ergeben sich aus der aktuellen Praxis des kognitiven Assessments von Patienten mit dem Leitsymptom Gedächtnisprobleme für die Versorgung und Frühintervention und welche Möglichkeiten ergeben sich aus dem Einsatz mobiler Apps?

Material und Methode. Auswertung aktueller Versorgungsstrukturen, Diskussion von Grundlagenarbeiten und Expertenempfehlungen und aktueller Entwicklungen.

Ergebnisse. Die aktuelle Praxis der „Paperpencil"-basierten und zeitlich sowie örtlich an die Praxis- und Klinikumgebung gebundenen Diagnostik kognitiver Defizite beeinträchtigt die Durchführbarkeit, Validität und Reliabilität des kognitiven Assessments und die Einschätzung der Progredienz kognitiver Defizite und limitiert so einen sinnvollen Einsatz für weiterführende diagnostische Maßnahmen und therapeutische Entscheidungen. Aktuelle Entwicklungen mobiler Apps, hier am Beispiel der neotiv-App exemplarisch dargestellt, können helfen, diese Probleme zu überwinden.

Schlussfolgerungen. Mobile App-basierte Technologien können helfen, die kognitive Diagnostik von Patienten mit dem Leitsymptom Gedächtnisprobleme zu verbessern. Sie können Über- und Unterversorgung reduzieren und einen gezielten und sinnvollen Einsatz weiterführender Diagnostik ermöglichen. Darüber hinaus können sie risikomodifizierende präventive Maßnahmen strukturieren, helfen iatrogene Ursachen kognitiver Probleme zu erkennen und in dieser Hinsicht auch die Patientenkompetenz stärken.

\section{Schlüsselwörter}

Alzheimer-Erkrankung · Leichte kognitive Beeinträchtigung · Mobile Apps . Neuropsychologie $\cdot$ Kognition

\section{Innovation in diagnostics-mobile technologies}

\section{Abstract}

Background. Progressive cognitive deficits are the main clinical symptom of Alzheimer's disease; however, the precise recording of cognitive deficits and assessment of their progression pose major problems in patient care and early interventions.

Objective. Which problems for care and early intervention result from the current practice of cognitive assessment of patients with memory problems and which opportunities arise from the use of mobile apps?

Material and methods. Evaluation of current care structures, discussion of basic work, expert recommendations and current developments.

Results. The current practice of the pencil and paper-based diagnostics of cognitive deficits, which is temporally and spatially bound to a clinical environment, constrains the feasibility, validity and reliability of cognitive assessment and the quantification of progression. This limits the meaningful use of further diagnostic measures, such as magnetic resonance imaging (MRI) and cerebrospinal fluid (CSF) analyses. Recent progress in mobile app-based technologies, illustrated here with the example of the neotiv app, can help to overcome these problems. Conclusion. Mobile app-based technologies can help to improve the cognitive assessment of patients with the main symptom of memory complaints. They can reduce overuse and underuse of diagnostic and therapeutic pathways and enable a targeted and meaningful use of advanced diagnostics. In addition, they can structure risk-modifying preventive measures, identify iatrogenic impairment of cognition and in this respect also strengthen patient competence.

Keywords

Alzheimer's disease - Mild cognitive impairment - Mobile apps · Neuropsychology . Cognition 
Beim Vorliegen eines SCI oder eines MCI kann die Kognition bei einem Teil der Betroffenen stabil bleiben. Beim Fehlen einer Progredienz können diagnostische Interventionen entsprechend reduziert werden. Auch bei Betroffenen mit einem positiven Alzheimer-Biomarkerprofil kann die prädementielle Phase sehr lange dauern (bis zu 20 Jahren; [13]) und hier ist es wichtig, reliabel zu erfassen, ob die Gedächtnisproblematik langsam oder schnell progredient ist und entsprechend zu beraten.

Diese Versorgungproblematik könnte durch innovative Tests, die eine definierte Beziehung zu Alzheimer-Biomarkern (Liquor-Tau oder -A $\beta$ ) haben und auBerhalb der ärztlichen Praxis oder der Klinik mittels mobiler Apps eingesetzt werden können, wesentlich verbessert werden. Darüber hinaus würde dies eine patientenindividuelle, hochfrequente, wiederholte und somit engmaschige Verlaufskontrolle erlauben. Eine vereinfachte und verbesserte Versorgung wird langfristig auch therapeutisch wichtig. Krankheitsmodifizierende Therapien der Alzheimer-Erkrankung werden nur wirksam sein, wenn sie in der präklinischen Demenzphase initiiert werden [29]. Hier erlauben innovative mobile Testverfahren perspektivisch kostengünstige Therapiestratifizierung und Therapiemonitoring.

\section{Frühintervention in der Zukunft}

Die präklinische Phase der AlzheimerErkrankung kann bis zu 20 Jahre andauern [13]. In der Zukunft müssen präventive Therapien möglichst in dieser präklinischen Phase einsetzen, bevor der irreversible Verlust neuronalen Gewebes klinisch relevante kognitive Probleme hervorruft [29]. Das bedeutet, dass in der Zukunft Interventionen zu einem Zeitpunkt initiiert werden, an dem die kognitiven Symptome noch subtil sind. Dieser Umstand misst der Erfassung leichter, spezifischer kognitiver Defizite, die schon in der präklinischen Phase progredient sind, eine besondere Bedeutung bei.

Hochfrequentes kognitives Monitoring wird in der Zukunft auch für eine personalisierte, Outcome-orientierte Behandlung an Bedeutung gewinnen. Die
Fortführung einer lang andauernden präventiven Behandlung ist nur sinnvoll, wenn die Progression der kognitiven Verschlechterung aufgehalten werden kann; das hierfür erforderliche kognitive Monitoring könnte durch mobile Apps effizient und reliabel gewährleistet werden. Aktuelle Studien zur Wirksamkeit von BACE (,beta-site amyloid precursor protein cleaving enzyme“) -Inhibitoren zeigen zudem, dass es auch von Bedeutung sein wird, potenzielle kognitive Nebenwirkungen von Interventionen frühzeitig zu erkennen [28].

\section{Vermeidbare Risiken, modifizierbare und iatrogene kognitive Störungen}

Eine große Herausforderung in der Versorgung älterer Menschen mit kognitiven Problemen ist die Unterscheidung iatrogener Ursachen kognitiver Störungen von einer biologischen Progression der Alzheimer-Erkrankung. Beispielsweise kann eine Reihe von Medikamenten kognitive Störungen induzieren [20]. Diese zu erkennen, wird in der aktuellen Praxis durch die Einschränkungen kognitiver Diagnostik erschwert. Hier kann das longitudinale Monitoring mit mobilen Apps eine wesentliche Verbesserung der Erkennung iatrogener, vermeidbarer Ursachen kognitiver Störungen ermöglichen.

Schließlich gewinnt eine individuelle und selbstmotivierte Reduktion von Risikofaktoren eine zunehmende Bedeutung in unserer älter werdenden Bevölkerung. Regelmäßige und sensitive kognitive Testungen können den Blick auf die eigene kognitive Gesundheit schärfen und dabei motivieren Risikofaktoren wie mangelnde Bewegung oder ungesunde Ernährung zu reduzieren [15].

\section{Vorteile App-basierter kognitiver Testungen}

Mobile Apps auf Endgeräten wie Smartphones oder Tabletcomputern erlauben die lebensnahe, hochfrequente und longitudinale Erfassung kognitiver Funktionen [12]. Damit lassen sich kognitive Funktionen unabhängig von den räumlich und zeitlich engen Vorgaben medizi- nischer Einrichtungen messen und verfolgen. Mit mobilen Apps können dabei kognitive Tests in Form kurzer spielerisch aufbereiteter Aufgaben in den Alltag integriert und regelmäßig absolviert werden [5]. Aktuelle Untersuchungen zeigen bereits eine Verfügbarkeit kompatibler Endgeräte von über $80 \%$ in der Altersgruppe der 55- bis 64-Jährigen [1] und diese Tendenz wird sich in den nächsten Jahren sicherlich beschleunigen. Organisatorisch kann eine App im eigenen Zuhause zu jeder Zeit eingesetzt werden und die Ergebnisse stehen dem Behandler zeitnah und automatisiert zur Verfügung. Es entfallen Terminvergaben und zeitintensive Analysen beim Haus-/Facharzt oder einer Klinik. Darüber hinaus entfällt die künstliche und stressreiche Untersuchungssituation sowie z. B. die Bereitstellung eines Raums für die Dauer der ungestörten Durchführung des Assessments. Hierdurch sinkt der Organisationsbedarf in der Routineversorgung und es wird eine Optimierung des Versorgungspfades erreicht, der letztendlich durch die kostengünstige Analyse auch die Vergütungsstruktur optimiert.

\section{) Mobile Apps können Versorgung und Frühintervention verbessern}

Durch den Einsatz mobiler Apps z.B. in der gewohnten Umgebung, meist dem eigenen Zuhause, können Faktoren, die falsch-positive Ergebnisse erzeugen, reduziert werden. Eine einzelne Testung, wie sie in der ärztlichen Praxis oder im Krankenhaus oft durchgeführt wird, muss nicht unbedingt die tatsächliche kognitive Leistung darstellen. Eine wiederholte Testung ist mit einer mobilen App alltagsnah und ohne wiederholte Arztbesuche möglich und kann die Messgenauigkeit erhöhen. Da Testungen über mehrere Tage verteilt werden können, entfällt auch die Notwendigkeit sehr langer und anstrengender Daueruntersuchungen. Da die Tagesform dennoch schwankt, erlauben mehrfach wiederholte Testungen eine Mittelung der Testergebnisse, um den Einfluss „schlechter“ Tage zu reduzieren 


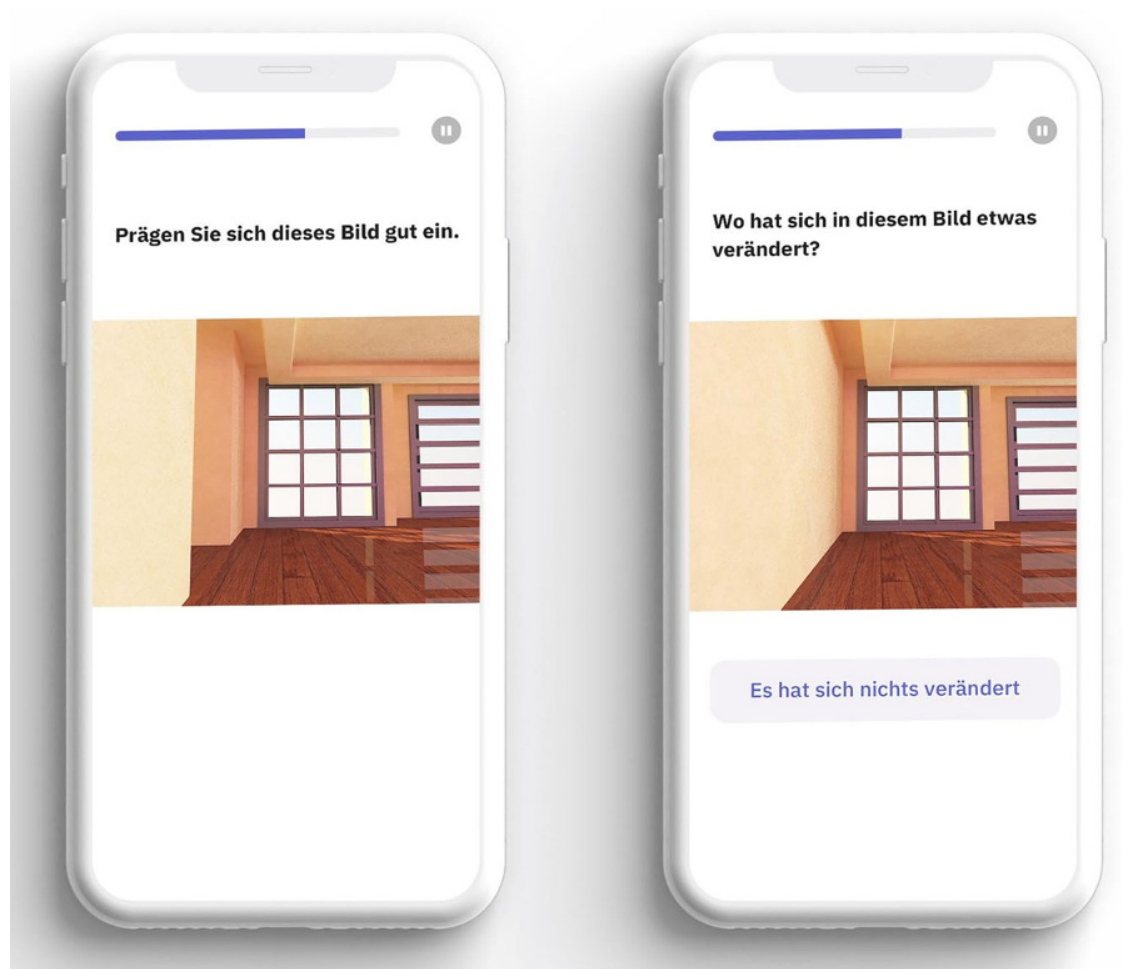

Abb. 1 A Beispielbildschirm eines Tests für Gedächtnispräzision für räumliche Szenen. Die Testperson muss sich verschiedene Bilder einprägen und anschließend bei einer erneuten Präsentation entscheiden, ob es sich um dasselbe oder ein leicht verändertes Bild handelt. Dabei tippt sie mit dem Finger an die Stelle des Raumes, die sich verändert hat (in diesem Fall auf die linke Wand). Falls die Testperson keine Veränderung erkennt, wird auf die Schaltfläche „Es hat sich nichts verändert" getippt. Die Leistung in diesem Test nimmt in der präklinischen und prodromalen Phase der Alzheimer-Erkrankung ab

und somit das Signal-Rausch-Verhältnis signifikant zu erhöhen [23].

Eine Verbesserung der Qualität der Identifikation und Diagnostik kognitiver Beeinträchtigungen durch mobile Apps kann zu einer adäquaten Behandlung und Beratung des Patienten beitragen. Die Rate der falsch-positiv als auch falsch-negativ Diagnostizierten kann sinken. Dies hätte direkte Auswirkungen auf Fehl-, Über- und Unterversorgung der Patienten. Ebenso können eine verbesserte diagnostische Sicherheit und die Kenntnis der tatsächlichen Progressionsrate zu einer besseren Beratung und zu mehr $\mathrm{Zu}$ friedenheit beim Patienten und dessen Behandler führen. Gegebenenfalls kann bei einer stabilen kognitiven Trajektorie frühzeitig Entwarnung gegeben werden. Eine verbesserte Früherkennung heißt perspektivisch auch eine frühere Intervention, mit dem Ziel einer Verzögerung und evtl. Stillstand des Voranschreitens der Erkrankung.

\section{Aktueller Entwicklungsstand}

Die Entwicklung geeigneter mobiler Apps erfordert eine enge Interaktion von Wissenschaftlern, Ärzten, Softwareentwicklern und Designern und eine direkte Translation von der Kognitions- und Alzheimer-Forschung in die Anwendung. Darüber hinaus muss die Entwicklung einer mobilen Plattform in Zusammenarbeit mit Zentren der Alzheimer-Forschung erfolgen. Ein Beispiel für eine solche Entwicklung ist die mobile App der neotiv ${ }^{\otimes}$ GmbH (Magdeburg, Deutschland), die in enger Kooperation mit dem Deutschen Zentrum für Neurodegenerative Erkrankungen und der Otto-von-Guericke-Universität Magdeburg erfolgt und bereits in internationalen Studien zum Einsatz kommt (- Abb. 1 und 2). Andere mobile sowie App-basierte Ansätze werden beispielsweise an der University of Washington St Louis in Zusammenarbeit mit der DIAN-Studie sowie an der University of Oxford entwickelt [18].
Eine mobile App für kognitives Monitoring im Kontext der Alzheimer-Erkrankung muss einen stringenten und langwierigen Entwicklungszyklus durchlaufen. Dieser Zyklus führt durch folgende Stadien (• Abb. 2):

a. Theoretische Fundierung des Untersuchungsansatzes:

Die theoretischen Konzepte der unterschiedlichen Testverfahren basieren auf aktuellen wissenschaftlichen Erkenntnissen.

b. Funktionelle Validierung der anatomischen Spezifizität:

Hirnaktivitätsmessungen zeigen Spezifität der Tests für Hirnregionen, die im Verlauf der Alzheimer-Erkrankung betroffen sind (im Fall von neotiv ${ }^{\circledast}$, Tests, die kognitive Funktionen von Hirnregionen erfassen, welche früh von Tau- und Amyloidpathologie betroffen sind Objektdiskrimination, Raumdiskrimination, Objekt-Raum-Assoziation, Konsolidierung).

c. Validierung der Testverfahren hinsichtlich Testgütekriterien:

Reliabilitäts- und Validitätsuntersuchungen bestätigen Testgütekriterien im Vergleich zu etablierten analogen Verfahren.

d. Validierung der Testsensitivität anhand traditioneller Biomarker: Forschungsstudien mit großen Kohorten quantifizieren die Beziehung zu molekularen Biomarkern der Alzheimer-Erkrankung.

e. Anpassung der Testverfahren an Smartphones und Tabletcomputer: Die Testverfahren werden zur Nutzung an Smartphones und Tabletcomputern adaptiert und mit adaptierten mobilen Versionen etablierter neuropsychologischer Tests kombiniert (im Fall von neotiv ${ }^{\circledR}$ mit TrailMaking-Test und Digit-Symbol-Substitution). Die Nutzbarkeit durch die Zielgruppe älterer Menschen und Patienten in präklinischen und prodromalen Erkrankungsstadien wird maximiert.

f. Datenschutzkonzept und Datensicherheit: Datenschutz- und Datensicherheitsaspekte müssen höchste Standards 


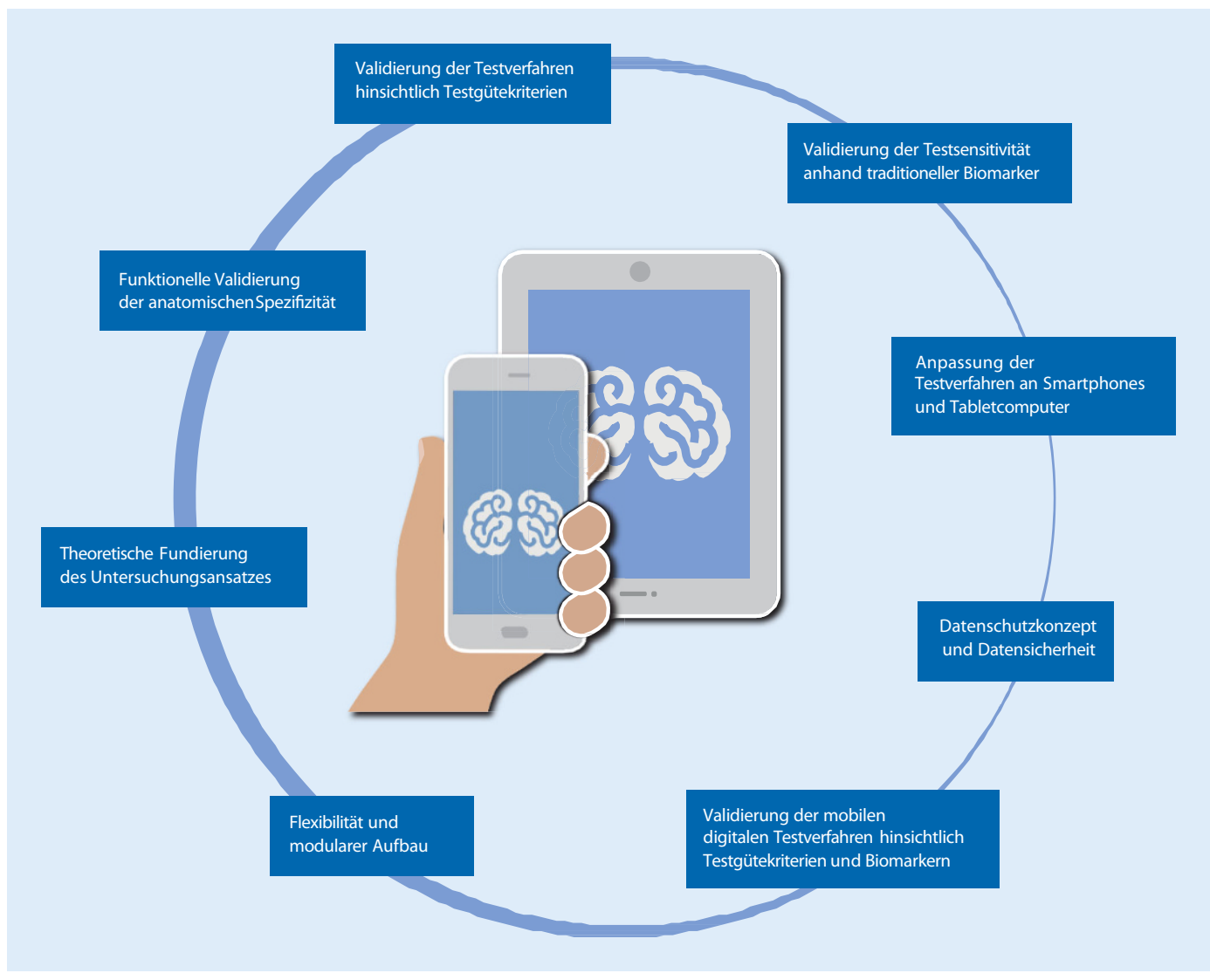

Abb. $2<$ Entwicklungsstadien einer mobilen Anwendung für Gedächtnistests am Beispiel der neotiv-App

mit entsprechenden Zertifizierungen durch autorisierte Stellen erfüllen.

g. Validierung der mobilen digitalen Testverfahren hinsichtlich Testgütekriterien und Biomarkern: Die als App implementierten Testverfahren bestätigen Testgütekriterien in Patientenkohorten.

h. Flexibilität und modularer Aufbau: Eine App-Plattform erlaubt eine flexible und adaptive Gestaltung der App für unterschiedliche klinische Anforderungen.

\section{Ausblick - Einsatz in der Routineversorgung}

Ein mögliches Szenario einer verbesserten Versorgung und Frühintervention mit mobilen Apps bei dem Leitsymptom Gedächtnisprobleme und der Verdachtsdiagnose einer Alzheimer-Erkrankung könnte in Zukunft folgendermaßen aussehen:

1. Bei Beschwerden über Gedächtnisprobleme wird nach Veranlassung des Hausarztes, Neurologen oder Psychiaters mit einer mobilen App gescreent (z. B. über einen Zeitraum von 6 Monaten). Dabei wird erfasst, ob das Gedächtnisprofil auf ein Risiko für eine Alzheimer-Erkrankung hinweist (im Sinne eines MCI) und ob die Trajektorie auf eine progrediente Verschlechterung hinweist.

2. Bei einer stabilen kognitiven Verlaufstrajektorie wird Entwarnung gegeben.

3. Bei einer progressiven kognitiven Verschlechterung wird eine weitergehende Diagnostik (Magnetresonanztomographie [MRT], ggf. Serummarker, Liquorpunktion oder Positronenemissionstomographie [PET]) durchgeführt.

4. Iatrogene Ursachen kognitiver Defizite werden berücksichtigt. Es erfolgt eine Beratung zur Reduktion von Risikofaktoren (z. B. metabolisch, kardiovaskulär) für eine Demenz und ggf. eine entsprechende Lebensstilumstellung.

5. Falls vorhanden, erfolgt eine präventive Behandlung mit einem kausal oder symptomatisch wirksamen Medikament.
6. Es folgt ein Monitoring des Therapieerfolgs und Coaching der Lebensstilumstellung und der Risikomodifikation mit der mobilen App (bei Vorhandensein einer pharmakologischen Intervention in der Zukunft auch im Sinne eines „drugcompanion").

Bei dem aktuellen Entwicklungsstand der Technologie könnte dieses Szenario in den nächsten Jahren realisiert werden.

\section{Fazit für die Praxis}

- Mobile, App-basierte Technologien können helfen, die Versorgung von Patienten mit Gedächtnisproblemen und der Verdachtsdiagnose der Alzheimer-Erkrankung substanziell zu verbessern.

- Erste Produkte stehen nach langwierigen Entwicklungszyklen kurz vor der Anwendungsreife.

- Mobile Apps können in Zukunft bei Beschwerden über Gedächtnisprobleme und der Verdachtsdiagnose 


\section{einer Alzheimer-Erkrankung zum} Einsatz kommen.

- Sie können durch hochfrequente Testungen in kurzer Zeit die Progredienz einer kognitiven Störung bestimmen.

- Eine weitergehende Diagnostik (MRT, ggf. Serummarker, Liquorpunktion oder PET) kann so auf die Fälle progredienter Störungen limitiert werden.

- latrogene Ursachen kognitiver Defizite können besser erkannt und eine Modifikation von Risikofaktoren besser strukturiert und monitoriert werden.

- Eine Lebensstilintervention oder eine personalisierte Therapie im Sinne eines Drug-companion-Ansatzes werden ermöglicht.

\section{Korrespondenzadresse}

\section{Prof. Dr. Emrah Düze}

Deutsches Zentrum für Neurodegenerative Erkrankungen (DZNE), Standort Magdeburg Leipziger Str. 44, 39120 Magdeburg,

Deutschland

Emrah.duezel@dzne.de

\section{Einhaltung ethischer Richtlinien}

Interessenkonflikt. E. Düzel und D. Berron sind Mitgründer der neotiv ${ }^{\circledR} \mathrm{GmbH}$. J.R. Thyrian gibt an, dass kein Interessenkonflikt besteht.

Für diesen Beitrag wurden von den Autoren keine Studien an Menschen oder Tieren durchgeführt. Für die aufgeführten Studien gelten die jeweils dort angegebenen ethischen Richtlinien.

Open Access. Dieser Artikel wird unter der Creative Commons Namensnennung 4.0 International Lizenz (http://creativecommons.org/licenses/by/4.0/deed. de) veröffentlicht, welche die Nutzung, Vervielfältigung, Bearbeitung, Verbreitung und Wiedergabe in jeglichem Medium und Format erlaubt, sofern Sie den/die ursprünglichen Autor(en) und die Quelle ordnungsgemäßnennen, einen Linkzur Creative Commons Lizenz beifügen und angeben, ob Änderungen vorgenommen wurden.

\section{Literatur}

1. Berenguer A, Goncalves J, Ferreira Detal (2017) Are smatphones ubiquotous? leee Consumer Electron Mag 17:2162-2248

2. Berron D, Neumann K, Maass A et al (2018) Agerelated functional changes in domain-specific medial temporal lobe pathways. Neurobiol Aging 65:86-97
3. Braak H, Del Tredici K (2015) The preclinical phase of the pathological process underlying sporadic Alzheimer's disease. Brain 138:2814-2833

4. Calamia M, Markon K, Tranel D (2012) Scoring higher the second time around: meta-analyses of practice effects in neuropsychological assessment. Clin Neuropsychol 26:543-570

5. Chinner A, Blane J, Lancaster C et al (2018) Digital technologies for the assessment of cognition: a clinical review. Evid Based Ment Health 21:67-71

6. Costa A, Bak T, Caffarra P et al (2017) The need for harmonisation and innovation of neuropsychological assessment in neurodegenerative dementias in Europe: consensus document of the Joint Program for Neurodegenerative Diseases Working Group. Alzheimers Res Ther 9:27

7. Dickerson BC, WolkDA (2010) Dysexecutive versus amnesic phenotypes of very mild Alzheimer's disease are associated with distinct clinical, genetic and cortical thinning characteristics. J Neurol Neurosurg Psychiatry. https://doi.org/10 1136/jnnp.2009.199505

8. Duzel E, Yonelinas AP, Mangun GR et al (1997) Event-related brain potential correlates of two states of conscious awareness in memory. Proc Natl Acad SciU SA 94:5973-5978

9. Eichler T, Thyrian JR, Hertel J et al (2015a) Rates of formal diagnosis of dementia in primary care: The effect of screening. Alzheimers Dement (amst) 1:87-93

10. EichlerT,Thyrian JR, Hertel Jetal (2015b) Subjective memory impairment: No suitable criteria for casefinding of dementia in primary care. Alzheimers Dement (amst) 1:179-186

11. Innocenti A, Cammisuli DM, Sgromo D, Franzoni F, Fusi J, Galetta F, Pruneti C (2017) Lifestyle, physica activity and cognitive functions: the impact on the scores of Montreal cognitive assessment (MoCa). Arch Ital Biol 155(1/2):25-32

12. InselTR(2017) Join the disruptors of health science. Nature 551(7678):23-26

13. Jack CR Jr., Bennett DA, Blennow K et al (2018) NIA-AA Research Framework: Toward a biological definition of Alzheimer's disease. Alzheimers Dement 14:535-562

14. Landy KM, Salmon DP, Filoteo JV et al (2015) Visual search in dementia with Lewy bodies and Alzheimer's disease. Cortex 73:228-239

15. Livingston S (2017) Bending the cost curve on high-risk patients. Mod Healthc 47:12-14

16. Maass A, Landau S, BakerSLetal (2017) Comparison of multiple tau-PET measures as biomarkers in aging and Alzheimer's disease. Neuroimage 157:448-463

17. Maass A, Lockhart SN, Harrison TM et al (2018) Entorhinal tau pathology, episodic memory decline, and neurodegeneration in aging. J Neurosci 38:530-543

18. Maass A, Berron D, Harrison TM et al Alzheimer's pathology targets distinct memory networks in the aging brain. Brain. https://doi.org/10.1093/ brain/awz154

19. Ossenkoppele R, Schonhaut DR, Schöll $M$ et al (2016) Tau PET patterns mirror clinical and neuroanatomical variability in Alzheimer's disease. Brain 139. https://doi.org/10.1093/brain/aww027

20. Redston MR, Hilmer SN, Mclachlan AJ et al (2018) Prevalence of potentially inappropriate medication use in older inpatients with and without cognitive impairment: a systematic review. J Alzheimers Dis 61:1639-1652

21. Scheurich A, Muller MJ, Siessmeier $T$ et al (2005) Validating the DemTect with 18-fluoro-2deoxy-glucose positron emission tomography as a sensitive neuropsychological screening test for early alzheimer disease in patients of a memory clinic. Dement Geriatr Cogn Disord 20:271-277

22. Schöll M, Lockhart SN, Schonhaut DR et al (2016) PET imaging of tau deposition in the aging human brain. Neuron 89:971-982

23. Sliwinski MJ, Mogle JA, Hyun J et al (2018) Reliability and validity of ambulatory cognitive assessments. Assessment 25:14-30

24. Strohmaier U, Keller F, Kilimann I et al (2018) Patients with dementia in primary care: who is referred to a neurologist/psychiatrist and what patient-oriented factors are associated with the visit? J Alzheimers Dis 64:925-932

25. Swanberg MM, Tractenberg RE, Mohs Ret al (2004) Executive dysfunction in Alzheimer disease. Arch Neurol 61:556-560

26. Thyrian JR, Hoffmann W (2012) Dementia care and general physicians - a survey on prevalence, means, attitudes and recommendations. Cent Eur J Public Health 20:270-275

27. Thyrian JR, Hoffmann W, Eichler T (2018) Editorial: early recognition of dementia in primary care-current issues and concepts. Curr Alzheimer Res 15:2-4

28. Vellas B, Aisen P, Weiner M et al (2018) What We Learn from the CTAD (Clinical Trials Alzheimer's Disease) 2018. J Prev Alzheimers Dis 5:214-215

29. Winblad B, Amouyel P, Andrieu S et al (2016) Defeating Alzheimer's disease and other dementias: a priority for European science and society. Lancet Neurol 15:455-532

30. Wolfsgruber $S$, Molinuevo $\mathrm{JL}$, Wagner $M$ et al (2019) Prevalence of abnormal Alzheimer's disease biomarkers in patients with subjective cognitive decline: cross-sectional comparison of three European memory clinic samples. Alzheimers Res Ther 11:8

31. Wong S, Bertoux M, Savage G et al (2016) Comparison of Prefrontal atrophy and episodic memory performance in Dysexecutive alzheimer's disease and behavioral-variant Frontotemporal dementia. J Alzheimer's Dis 51:889-903

32. Woodward MC, Rowe CC, Jones G et al (2015) Differentiating the frontal presentation of Alzheimer's disease with FDG-PET. J Alzheimer's Dis 44:233-242 